\title{
Recent Progress on CVD Growth of Graphene from a Liquid Carbon Precursor
}

\author{
Azzafeerah Mahyuddin ${ }^{a, b, *}$, Abd. Khamim Ismail ${ }^{a}$, Muhammad Firdaus \\ Omara $^{a}$, Ainul Hakimah Karim ${ }^{\mathrm{b}}$
}

a Department of Physics, Faculty of Science, Universiti Teknologi Malaysia, 81310 Johor, Malaysia; ${ }^{b}$ Department of Quality Engineering, Universiti Kuala Lumpur, Malaysian Institute of Industrial Technology, Persiaran Sinaran IImu, Bandar Seri Alam, 81750 Johor, Malaysia.

\begin{abstract}
Graphene has become a remarkable highlight of advanced material research because of its far superior properties compared to other materials. Chemical vapor deposition (CVD) has emerged as an essential method for scalable production and large area graphene for various applications. Various carbon precursors have been reported for graphene production as they can dramatically impact the graphene growth yield. In the early years of graphene CVD growth, hydrocarbon gases such as methane and acetylene have become favorable carbon precursors because of their stability at elevated temperature and controllable growth. However, hydrocarbon gases are known as explosives and toxic, therefore require a growth system with a high degree of safety and handling precautions. With the limitations mentioned above, liquid carbon source may change the graphene growth landscape as it is relatively inexpensive, nonexplosive compared to the conventional gaseous precursor. This article aims to review a detailed synthesis of large-area graphene using liquid carbon precursors via the CVD technique. Challenges and future perspectives are highlights.
\end{abstract}

Keywords: Graphene, Chemical vapor deposition, Liquid precursor, Synthesis method

*For correspondence: azzafeerah@unikl.edu.my

Received: 8 Nov 2020

Accepted: 2 June 2021

(C) Copyright Mahyuddin. This article is distributed under the terms of the Creative Commons Attribution License, which permits unrestricted use and redistribution provided that the original author and source are credited.

\section{Introduction}

Graphene is a two-dimensional carbon allotrope consists of a one-atom thick planar sheet of $\mathrm{sp}^{2}$ bonded carbon atoms densely packed in a honeycomb crystal lattice. Since the first fabrication of isolated samples by mechanical exfoliation of graphite requires a time-consuming process and small graphene yields, the chemical vapor deposition (CVD) technique is favored to provide large area coverage. CVD is a process that exposes the substrate or catalyst to carbon precursors, which will react and/or decompose on the substrate surface to create the graphene film deposit. The development of a large area of high-quality graphene on catalytic metal or non-metallic substrates had created researchers' significant interest. Hydrocarbons and polymers with $\mathrm{C}$ - and $\mathrm{H}$-based compounds are commonly used for graphene synthesis.

Most of the published works aimed to highlight the control of domain size and the number of graphene layers. The density of grain boundaries and defects of the synthesized graphene-based materials also becomes significant debates. Up until now, a limited review has been done for CVD growth of graphene, specifically by using liquid carbon precursor. This review will provide an overview of recent progress, 
efforts, and challenges in the graphene production using the CVD method. The first part of the paper will discuss graphene properties and their application. The second part is the core section, which will discuss recent progress in synthesizing graphene from CVD. This core section will be divided into part one on graphene synthesis using liquid and organic carbon precursor and the second part will discuss some suggestions for future research. This review will give a researcher a better understanding of graphene growth using liquid precursors as a carbon source in the CVD technique.

Graphene is a single atom thick sheet of $\mathrm{sp}^{2}$ hybridized carbon atoms arranged in a honeycomb lattice structure. Its outstanding properties include high surface area, high electrical conductivity, good chemical stability, and strong mechanical strength. The popularity of graphene in scientific research was pioneered by Noble laureates Prof. Andre K. Geim and Prof. Konstantin Novoselov [1]. Since their discovery, graphene has attracted researchers in every sector from energy, environment, and health.

Graphene can be prepared by several methods, which can be categories as a bottom-up approach and a top-down approach. Pyrolysis, epitaxial growth, chemical vapor deposition (CVD), and plasma synthesis are categories as a bottom-up approach. In contrast, the top-down approach includes mechanical exfoliation, chemical exfoliation, and chemical fabrication. Among the above synthesis methods, CVD has been widely studied as the as-produced graphene can be grown over a large area and uniform film. Various metal catalysts such as $\mathrm{Cu}{ }^{[2][3][4]}, \mathrm{Ni}{ }^{[5][6]}, \mathrm{Pt}{ }^{[7][8]}, \mathrm{Ru}{ }^{[9],}$ and $\mathrm{Ir}{ }^{[10]}$ have been proven to ease the graphene growth. The catalyst was reported to control type, thickness, crystal orientation, and size area of the crystal growth [11]. Seah et al. have reviewed the fundamental mechanism of CVD graphene-growth on transition metal ${ }^{[12]}$. Among the reported catalyst, $\mathrm{Cu}$ has been widely used due to its low carbon solubility and good control graphene growth. Due to its low carbon solubility, single-layer graphene (SLG) is often reported for graphene yield.

Meanwhile, $\mathrm{Ni}$ is a catalyst that exhibits high carbon solubility, which results in multilayer graphene (MLG). Interestingly, a few researchers reported the number of graphene layers could be controlled by adjusting the gas flow rate, reaction time, and cooling rate [13][14][15]. Ni is seen to have a good performance at a low-temperature catalyst, overcoming the Cu catalyst limitations associated with high growth temperature, usually greater than $1000^{\circ} \mathrm{C}$.

CVD paves the way for graphene growth onto targeted metal catalyst and is a comparatively reliable and feasible method for industrial applications. However, conventional CVD uses purified gaseous precursors such as $\mathrm{CH}_{4}$ and $\mathrm{C}_{2} \mathrm{H}_{2}$, known as explosive and expensive. Moreover, high growth temperature impedes the development of practical graphene-based devices for everyday use.

\section{The CVD technique with liquid precursors}

\section{Ethanol}

Despite the conventional CVD method of methane, few groups have successfully demonstrated ethanol as a precursor to synthesizing graphene. Miyata et al. demonstrated graphene growth using flash cooling methods after the CVD process ${ }^{[16]}$. Single graphene was successfully grown on Ni foil. Raman spectra reveal a $G$ band at $1580 \mathrm{~cm}^{-1}$ with a narrow full width of half maximum (FWHM) of $33 \mathrm{~cm}^{-1}$, which corresponds to single-layer graphene. The low intensity of $D$ peak and high $G / D$ ratio suggests the graphene possesses high quality and its grain size is larger than $500 \mathrm{~nm}$, respectively. Raman mapping reveals the grain size of single-layer graphene in the range of 1 to $10 \mu \mathrm{m}$. Interestingly this study indicates no graphene was observed during naturally cooled substrates of CVD growth. However, the flash cooling method leads to graphene formation and does not lead to decomposition like the natural cooling process. As many works focused on conventional precursor with a low-pressure CVD method, ethanol grown graphene with atmospheric pressure CVD is reported to exhibit $50-60 \%$ single and double layer graphene yield ${ }^{[17]}$. It is interesting to notice the Raman spectra display $G$ and $2 D$ peaks at $\sim 1575$ and $\sim 2700 \mathrm{~cm}^{-1}$ for all different thickness graphene films, which is agreeing with the literature. $I_{2 D} / I_{G}$ and $I_{D}$ $/ \mathrm{I}_{\mathrm{G}}$ ratio are 2.10 and 0.23 , respectively. The full-width at half maximum (FWHM) of the 2D band is determined to be $\sim 20.50 \mathrm{~cm}^{-1}$. This work is also intended to compare the characteristics of graphene 
grown from ethanol and pentane. Interestingly, ethanol-grown graphene has lower defect density, better hexagonal carbon lattice, and better uniformity compared to pentane-grown. These findings can be seen from Raman studies. Although, FWHM of the 2D band from graphene-pentane is $\sim 20.80 \mathrm{~cm}^{-1}$, its $\mathrm{D}$ band at $\sim 1350 \mathrm{~cm}^{-1}$ is more noticeable. Moreover, there is a weak $D^{\prime}$ peak at $\sim 1630 \mathrm{~cm}^{-1}$ and $\mathrm{I}_{\mathrm{D}} / \mathrm{I}_{\mathrm{G}}$ ratio is around 0.66 , which is bigger than that of graphene grown by ethanol. The measured sheet resistance and hall mobility of graphene from ethanol precursor can reach $2657 \Omega / s q$ and $110 \mathrm{~cm}^{2} / \mathrm{Vs}$, respectively. Besides, a comparison between two different alcohols, 2-phenylethanol, and ethanol, has also been studied by Campos-Delgado et al. ${ }^{[18]}$. The results suggest that monolayer graphene was produced by using both precursors. However, graphene grown ethanol produced domains of larger $10 \times 10 \mu \mathrm{m}$ single layer, while 2-phenylethanol gives an average of $2 \times 2 \mu \mathrm{m}$ islands grain. The FWHM of the G' band for both precursors is about $30-36 \mathrm{~cm}^{-1}$ and $\mathrm{I}_{\mathrm{G}} / \mathrm{I}_{\mathrm{G}}$ ratio is $\sim 3$. The optimal growth conditions for ethanol as a precursor are $980^{\circ} \mathrm{C}$ for $10 \mathrm{~s}$ with a flow rate of $1 \mathrm{l} / \mathrm{min}$.

Meanwhile, several groups report pre-oxidation treatment of $\mathrm{Cu}$ foil, which acts as a metal catalyst. Chen et al. used to heat the $\mathrm{Cu}$ foil on a hot plate of $250^{\circ} \mathrm{C}$ temperature prior to the CVD process ${ }^{\text {[19]. The foil }}$ surface is then changed from shiny red into a dull gray. They proved a clear dependence between preoxidation duration and nucleation density of graphene domains. Interestingly, the nucleation density can reach up to $\sim 0.1 \mathrm{~mm}^{-2}$ with 90 min pre-oxidation treatment compared to those without the pre-oxidation process, which observed nucleation density only at $\sim 8 \mathrm{~mm}^{-2}$. In addition, Gnisci et al. proposed that the large nucleation density induced by ethanol-CVD restricted the growth of grains larger than 1-3 $\mu \mathrm{m}$ without $\mathrm{Cu}$ pre-oxidation ${ }^{[20]}$. A integration of $\mathrm{Cu}$ pre-oxidation, quick Ar annealing, and low ethanol flow rate during CVD growth are the graphene growth key parameters of isolated large grains on the plain catalyst. By optimizing these parameters, a few nucleation sites build, and ethanol-dissociated carbon atoms ideally integrate into the growing nuclei instead of further promoting to nucleation. These findings also pointed pre-oxidation treatment was critical in preventing nucleation density. Besides, Raman analysis revealed the graphene films is monolayer with $I_{2 D} / I_{G}$ is 2.3 . The field-effect mobility surpassing $1300 \mathrm{~cm}^{2} / \mathrm{Vs}$ and sheet resistance is in the range of $550-610 \Omega / \mathrm{sq}$.

A simple substrate such as stainless steel has been reported by John et al. to grow graphene using the thermal CVD method ${ }^{[21]}$. This group was successfully synthesized from single to a few layers of graphene over a large surface area. This work is intended to develop a new catalytic metal for graphene growth. The $I_{G} / I_{2 D}$ ratio of $\sim 0.7$ and $F W H M$ of $\sim 32 \mathrm{~cm}^{-1}$ at the $2 \mathrm{D}$ band from Raman spectrum shows this substrate can produce single graphene. Despite that, Raman spectroscopic study with a combination with XPS analysis indicates some oxide species such as $\mathrm{Mn}$ and $\mathrm{Cr}$ play a crucial role in the non-homogeneity of the formed graphene. This knowledge of the surface chemical species might be a new insight in preparing graphene on stainless steel.

The growth of graphene from ethanol remains a challenge to produce large crystal grains. Many researchers agreed that growing single-crystal grains with minimal defects in flat metal catalytic surfaces is very critical for technology applications. The usage of different catalyst to grow graphene offers many opportunities for further studies. Graphene grown ethanol still need to be transferred onto desired substrates. The formation of wrinkles due to thermal stress because of wide difference in thermal expansion between graphene and $\mathrm{Cu}$ substrate remains a huge challenge.

\section{Hexane}

Hexane is reported to be a novel liquid precursor. It has advantages over the gas phase, such as methane and ethylene. This organic compound and its derivatives found in the liquid phase at room temperature are cheap and readily available. Graphene grown from hexane liquid was first reported by Srivastava et al. ${ }^{22]}$. The graphene layer possesses monolayer and double layers observed by Raman spectroscopy, thus confirming that the films grown using vacuum-assisted chemical vapor deposition (VA-CVD) are acceptable. The FWHM of 2D peaks for monolayer is $40 \mathrm{~cm}^{-1}$ while $43 \mathrm{~cm}^{-1}$ for double layers graphene. Graphene synthesized from hexane shows good electrical conductivity with a typical sheet resistance of $100-300 \Omega / s q-c m$. In addition, the use of Cu foil as a catalyst is a good choice for the researcher to synthesize graphene from hexane liquid. Prior to graphene growth via CVD, a group reported that the $\mathrm{Cu}$ foils were fully submerged in a ferric nitrate solution to polish the surface for 2.5 
hours $^{[23]}$. The catalyst substrate's polishing process is necessary and proven to grow graphene onto a smooth substrate easily. The homogeneous graphene layers result in $74.8 \%$ in transmittance and $2.3 \%$ of absorbance per layer. The usage of hexane in the fabrication of graphene is still in the early stage. Therefore, more investigation is needed to increase the literature. Moreover, hexane in the liquid phase may help in realizing graphene doping.

\section{Benzene}

There is a remarkable interest in synthesizing large-area graphene at a low temperature, typically using CVD techniques. Benzene as a carbon feedstock is reported to be the best candidate for lowtemperature growth. Graphene flakes were first reported at a low growth temperature of around $300^{\circ} \mathrm{C}$ using low-pressure CVD ${ }^{[24]}$. Surprisingly, uniform monolayer graphene was achieved at this low temperature with FWHM of 2D peak is $28 \mathrm{~cm}^{-1}$. Since then, many works have focused on low pressure of approximately 8 and 15 Torr for benzene-grown graphene. However, Kang et al. was first demonstrated the atmospheric pressure CVD (APCVD) graphene ${ }^{[25]}$. The fabricated graphene possesses monolayer flakes with excellent quality, even growth temperature as low as $300^{\circ} \mathrm{C}$ in atmospheric ambient. A low degree of defect is confirmed from the $\mathrm{I}_{D} / \mathrm{I}_{\mathrm{G}}$ ratio of $\sim 0.35$ and automatically highlights the monolayer graphene domains possessed high uniformity structure. Raman spectra reveal $\mathrm{I}_{2 \mathrm{D}} / \mathrm{I}_{\mathrm{G}}$ ratio for multilayer domains is 0.78 and bilayers domains is 1.17 . Increasing $\mathrm{H}_{2}$ gas flow rate during the graphene growth resulted in larger domain sizes due to the increased growth rate. Moreover, altering the benzene quantity and growth time can determine the number of layers and graphene domains.

Jang et al. reported that the graphene growth routes implemented in low-pressure conditions might experience severe evaporation of metal catalysts, leading to a deterioration of graphene quality ${ }^{[26]}$. Moreover, to obtain high-quality graphene, oxygen, and water in ambient air should be eluded. Therefore, this group demonstrated a modified APCVD route named 'oxygen-free APCVD' process to synthesize graphene onto $\mathrm{Cu}$ foils. With this method, the lowest temperature for graphene growth is reported to as low as $100^{\circ} \mathrm{C}$. Figure 1 shows schematics diagrams of graphene's growth mechanisms on a Cu surface by LPCVD, normal APCVD, and oxygen-free APCVD. They showed the graphene films are continuously grown using an oxygen-free APCVD method because of the absence of residual oxidizing species compared to LPCVD and normal APCVD. The synthesized graphene grown at $300^{\circ} \mathrm{C}$ possessed a monolayer structure with a surface coverage of $100 \%$. Moreover, the graphene transmittance and carrier mobilities are $97.6 \%$ and $1,900-2,500 \mathrm{~cm}^{2} \mathrm{~V}^{-1} \mathrm{~s}^{-1}$, respectively. Raman measurement indicates monolayer graphene showed uniform $I_{2 D} / G(>1.8)$ and a small value of $I_{D} / G(<0.2)$. Moreover, the sheet resistance was $\sim 1,000 \Omega / \mathrm{sq}$ which is much outstanding than those of lowtemperature graphene from the literature ${ }^{[27]}$. Consequently, this new route is a notable step toward graphene's direct growth for electronic applications and flexible devices.

To consider several factors for synthesizing graphene by using the CVD process, Shanmugam's group studied various effects on the number of graphene layers and its crystallinity, for instance, temperature, type of precursor, and carbon precursor flow rate ${ }^{[28]}$. Data from the experimental statistical design (DoE) method is used to understand the CVD mechanism for synthesizing graphene from this aromatic carbon precursor. From the experiment, high carbon flow rates will yield a predominantly bilayer than a single layer. However, single-layer graphene for benzene may achieve at low pressure with a temperature of $1000^{\circ} \mathrm{C}$. This study is intended to propose a possible mechanism of single-layer graphene from the aromatic precursor. Two possible mechanisms of graphene growth are presented. At low decomposition temperature $\left(\sim 800^{\circ} \mathrm{C}\right)$, the radical species are likely aromatic, whereas, at high decomposition temperature $\left(\sim 1000^{\circ} \mathrm{C}\right)$, the ring is opened. Thus, the number of smaller radicals increased. Active radicals are then deposited on the copper catalyst surface. Then radicals undergo a copper surfacediffusion process. Consequently, the radicals will be dehydrogenated and cyclized into 'benzene' rings. At the final stage, the copper surface radicals are stabilized by secondary bonding interactions. Subsequently, covalent bonding between these radicals will take place and thus grow into a graphene sheet. 
(a) LPCVD

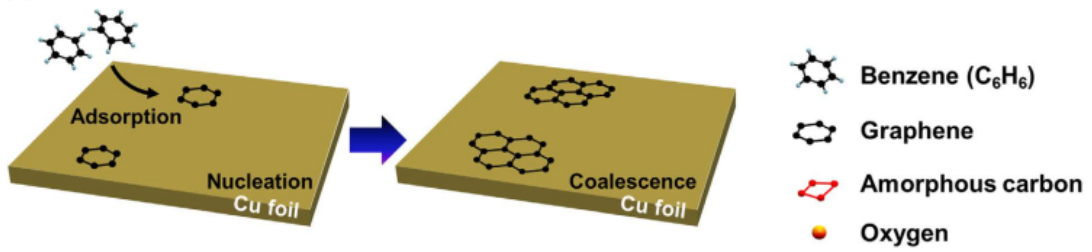

(b) Normal APCVD

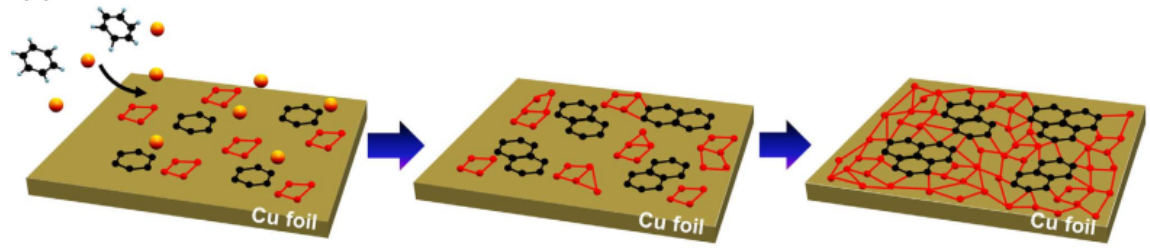

(c) Oxygen-free APCVD

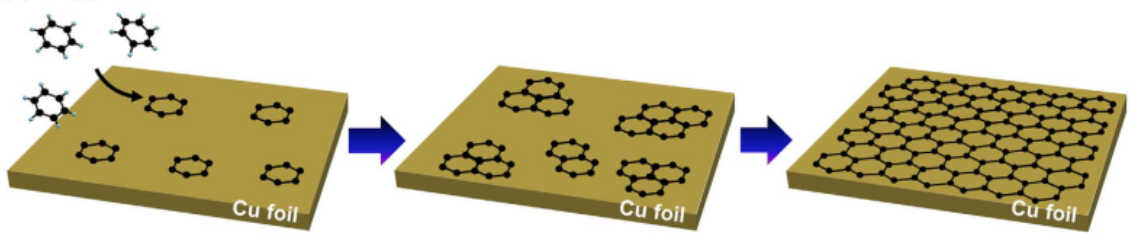

Figure 1. Schematic diagrams showing graphene growth mechanism on a Cu surface by (a) LPCVD, (b) normal APCVD, and (c) oxygenfree APCVD. Adapted from ref. [26]

The usage of $\mathrm{Cu}$ foils as a catalyst has become favored to synthesis graphene from benzene. However, most of the literature reported that graphene thin film derived from benzene still suffered from wrinkles during graphene transfer onto targeted substrates. Despite the wrinkle problem, benzene residues and vacancy defects in benzene-derived graphene at low temperatures even occurred. Nevertheless, the CVD growth temperature of $900-1000^{\circ} \mathrm{C}$ may be one of the alternatives to minimize the vacancies and defects ${ }^{[29]}$. The average ratio of $\mathrm{I}_{2 \mathrm{D}} / \mathrm{I}_{\mathrm{G}}$ for a $1000^{\circ} \mathrm{C}$ grown sample is 1.4 compared to a $900^{\circ} \mathrm{C}$ sample which is 2.35 . Furthermore, defect density which can be obtained from $I_{D} / I_{G}$ ratio is 0.08 for the $1000^{\circ} \mathrm{C}$ sample, whereas, with a decrease in temperature, the value of $I_{D} / I_{G}$ is increasing (i.e 0.14 and 0.35 at 900 and $825^{\circ} \mathrm{C}$, respectively). This result can be explained by the behavior of benzene molecules at a high temperature which can automatically degrade and self-dehydrogenate yielding carbon radicals. These carbon radicals, along with those produced by partial dehydrogenation of benzene on $\mathrm{Cu}$, are projected to act as graphene precursors. Because $\mathrm{Cu}$ has a low carbon solubility at high temperatures, graphene nucleation and growth are expected to occur on the $\mathrm{Cu}$ surface. The production of carbon radicals in the gas phase because of impulsive benzene degradation could speed up graphene nucleation and growth.

The difficulty of maintaining good properties for benzene-growth graphene remains a challenge. A detailed understanding of the graphene growth mechanism is very crucial for practical applications. Moreover, it is also important to consider alternative metal catalysts for benzene feedstock for literature. Furthermore, a transfer-free process for graphene films needs to be considered for device integration. This process often suffers from impurities and defects that become unfavorable for nano optoelectronic applications.

\section{Toluene}

Toluene, another type of aromatic hydrocarbon, has become of recent interest by a few researchers. For instance, continuous monolayer graphene films were successfully grown on electropolish Cu foils by the LPCVD system ${ }^{[30]}$. The flat electropolished $\mathrm{Cu}$ foils were proven to ease the growth process, which results in larger graphene domains. Toluene is identical to graphene's basic unit with a ring-structured molecular body. In addition, toluene is strongly absorbed on metal surfaces and has low $\mathrm{C}-\mathrm{H}$ bond 
dissociation energy, enabling easier dehydrogenation and tenfold higher carbon growth. Raman spectra reveal monolayer graphene features with FWHM of $2 \mathrm{D}$ peak is $\sim 39 \mathrm{~cm}^{-1}$ and $\mathrm{I}_{\mathrm{G}} / \mathrm{I}_{2 \mathrm{D}}$ is $\sim 0.5$. in addition, Raman maps of the intensity ratio of $I_{D} / I_{G}$ show values of less than $\sim 0.85$. The transmittance of the graphene film at $550 \mathrm{~nm}$ wavelength is $97.33 \%$ and sheet resistance is $\sim 8.02 \times 10^{3} \Omega / \mathrm{sq}$. High sheet resistance may result from the increased grain boundaries and defects of the graphene. Kim et al. study the differences in the growth process between toluene and xylene on semi-insulating SiC with the CVD system without using any metal substrates [31]. From the Raman spectra, the toluene-generated graphene showed high-intensity D and G peaks, confirming some graphene layers with some defects. However, graphene-grown xylene had a typical GO Raman spectrum with prominent D, G, and 2D peaks. $D$ peak is the prominent peak of $G O$ generated from hydroxyl and epoxide groups on the carbon basal plane. High-resolution transmission electron microscopy (HR-TEM) study for toluene-based graphene reveals carbon atoms were assembled in a hexagonal structure. The selected area energy diffraction (SAED) pattern was similar to the SAED pattern of highly ordered pyrolytic graphite indexed as the [001] zone axis. Whereas SAED pattern for xylene-based graphene showed a ring share which indicates an amorphous structure of $\mathrm{GO}$. It was produced by distortion of $\mathrm{sp}^{3} \mathrm{C}-\mathrm{O}$ bonds. The electrical conductivity of toluene-graphene was $30 \mathrm{~S} \mathrm{~cm}^{-1}$ at room temperature and reached $162 \mathrm{~S} \mathrm{~cm}^{-1}$ after undergone heat treatment at $300^{\circ} \mathrm{C}$. Toluene is comparable to the basic unit of graphene with its ringstructured molecular body. Likewise, in comparison to benzene, toluene is strongly absorbed on metal surfaces and has a low $\mathrm{CH}$ bond dissociation energy, allowing for easier dehydrogenation and a tenfold increase in carbon growth rate. Since the use of toluene for graphene research and development is still new, additional experimental work should be performed to understand the routes of actual precursor decomposition.

\section{Alternative carbon precursor}

Apart from liquid carbon feedstock, a more sustainable precursor has been tested for graphene synthesis. The following section reported methods to synthesized graphene from liquid biomass carbonbased. Graphene has been synthesized from soybean oil using a single step, rapid thermal synthesis in an ambient-air environment without any compressed gases ${ }^{[32]}$. The synthesized graphene has excellent, tunable film properties and is equivalent to conventionally synthesized graphene. This ambient-air process potentially addressed critical roadblocks such as lengthy high annealing temperature for the metal catalyst. It is also critical to use purified and compressed gasses to transport carbon-source materials and prolonged vacuum activity to prevent any adverse reactive oxygen species from the air.

Figure 2 shows the process of growing graphene film on Ni polycrystalline by using soybean oil. The Raman spectra indicated that at $800^{\circ} \mathrm{C}$ of growth temperature, single to few layers' graphene were successfully grown on the Ni foil. This work also showed that a rapid cooling method enabled graphene film to segregate and continuously grow on the substrate. On average, the graphene film exhibits $93.9 \%$ of optical transmission, $324 \Omega \mathrm{sq}^{-1}$ of sheet resistance, $0.15-0.25$ of Raman $\mathrm{I}_{\mathrm{D}} / \mathrm{I}_{\mathrm{G}}$ ratio, and $0.95-1.50$ $\mathrm{I}_{2 \mathrm{D}} / \mathrm{I}_{\mathrm{G}}$ ratio. From TEM analysis, the graphene film is comprised of domains spanning $\sim 200-500 \mathrm{~nm}$. Furthermore, HR-TEM images at the domain edges indicate the few-layered graphene domains in the interior of the film. Interestingly, the graphene films synthesized from soybean oil possess carrier mobility and average sheet resistance of $500-750 \mathrm{~cm}^{2} \mathrm{~V}^{-1} \mathrm{~s}^{-1}$ and $324 \Omega \mathrm{sq}^{-1}$, respectively. Moreover, as confirmed by X-ray photoelectron spectroscopy (XPS), the synthesized graphene film has a good $\mathrm{sp}^{2} / \mathrm{sp}^{3}$ ratio of $\sim 5.0$ and comparable with those generated by the conventional CVD method.

Graphene growth technology precursors have a critical role in determining the graphene output. As mentioned above, precursors from non-renewable sources are predominantly toxic and potentially explosive. To attempts simple green processes, Melaleuca alternifolia oil, obtained from distillation teatree leaves, was reported to synthesized graphene [33], [34]. It's hydrocarbon-rich, with over 100 compounds. The main component of volatile extract is terpinene-4-ol $\left(\mathrm{C}_{10} \mathrm{H}_{16}, 5-13 \%\right)$, $\mathrm{Y}$-terpinene $\left(\mathrm{C}_{10} \mathrm{H}_{16}, 10-28 \%\right)$, a-terpinene $\left(\mathrm{C}_{10} \mathrm{H}_{16}, 5-13 \%\right)$, and 1,8-cineole $\left(\mathrm{C}_{10} \mathrm{H}_{18} \mathrm{O}, 0-15 \%\right)$. Ouyang et al. reported to synthesized vertical graphene sheet (VGS) by using a homemade plasma-enhanced chemical vapor deposition (PECVD) system ${ }^{[34]}$. Porous Ni foam was used as a catalyst substrate. The Raman spectra suggest a nanocrystalline architecture of the graphene sheet in a large number of defects and edges. The ratio of $\mathrm{I}_{\mathrm{D}} / \mathrm{I}_{\mathrm{G}}$ is around $1.05-1.59$ and the ratio of $\mathrm{I}_{2 \mathrm{D}} / \mathrm{I}_{\mathrm{G}}$ is estimated around $0.17-$ 0.21 . The fabricated graphene material is suitable for next-generation high-performance supercapacitors 
with specific capacitance retention of $54 \%$. In addition, the specific capacitance of the graphene is 311 and $168 \mathrm{Fg}^{-1}$ at 1 and $20 \mathrm{Ag}^{-1}$, respectively.

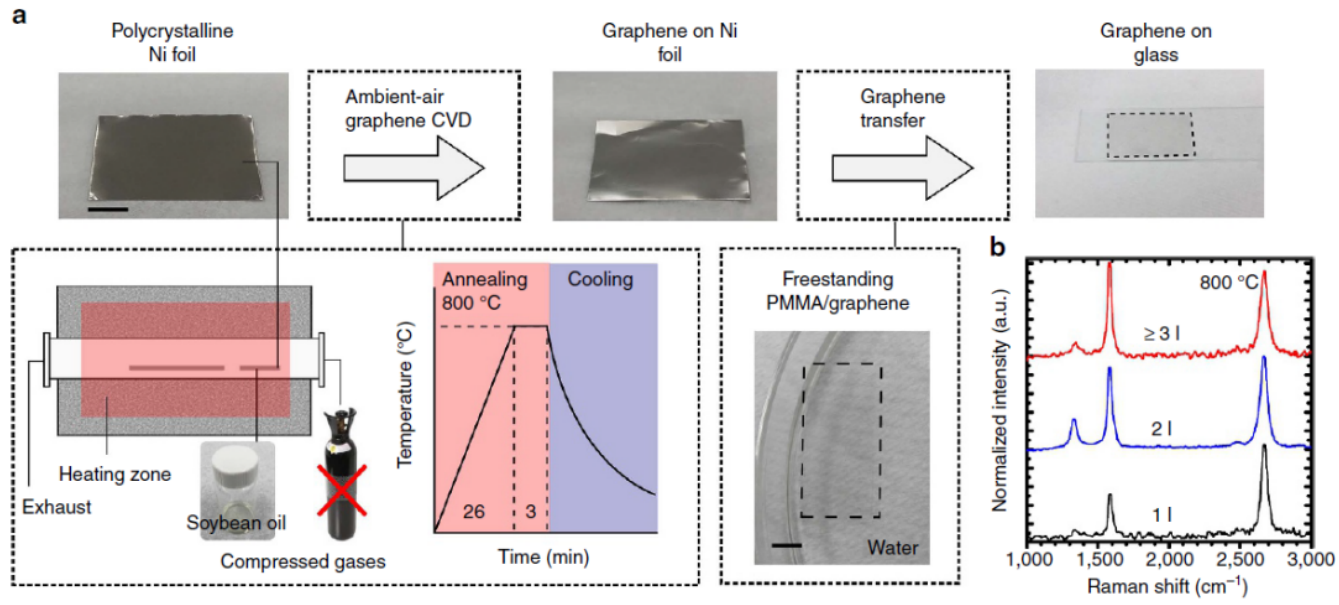

Figure 2. Growing graphene films in ambient air. (a) Polycrystalline Ni foil is thermally annealed with soybean oil precursor, and controlled graphene synthesis is promoted in the ambient air environment. Graphene films are being transferred to glass substrate. (b) Raman spectra suggest 1-layer, 2-layer and $>3$-layer regions in $800^{\circ} \mathrm{C}$ graphene. Adapted from [32]

Meanwhile, Jacob et al. deposit graphene by PECVD in the non-existence of a catalyst ${ }^{[33]}$. $\mathrm{SiO}_{2} / \mathrm{Si} \mathrm{has}$ used a substrate and pretreated with plasma for $1 \mathrm{~min}$ in $800^{\circ} \mathrm{C}$ ambient temperature. Since M.'s. Alternifolia is volatile at ambient temperature, no vapor heating was necessary in the chamber. This process was claimed to be quick, sustainable, scalable, and cost-effective. Graphene consists of hybridized carbon $\mathrm{sp}^{2}$ with traces of carbon $\mathrm{sp}^{3}$ and $\mathrm{C}-\mathrm{O}$ bonds. From Raman studies, the films were nearly defect-free. FWHM of the $2 \mathrm{D}$ peak showed approximately $60 \mathrm{~cm}^{-1}$. The $\mathrm{I}_{\mathrm{D}} / \mathrm{I}_{\mathrm{G}}$ ratio is 0.63 indicating low defect density of graphene film and primarily comprised of $\mathrm{sp}^{2}$ hybridized carbon. The appearance of the D' peak is visible for samples prepared at a duration of up to 2 min. Therefore, the ratio of $I_{D} / I_{D}$, are $2.75,4.8$, and 1.7 for $1 \mathrm{~s}, 1 \mathrm{~min}$, and 2 min samples, respectively. As an indicator, the value of $I_{D} / I_{D}$ above 13 shows graphene with high sp ${ }^{3}$ defects, 7 indicates vacancies, and 5 is boundary defects. Furthermore, even though the original precursor was multicomponent, no impurities were observed during the XPS studies. Only carbon C 1s peak is detected at about $284 \mathrm{eV}$. Apart from that, the transmittance of these films yielded a contact angle of $135^{\circ}$ with more than $85 \%$. The films have also proven to be a promising feature in resistive random-access memory (RRAM).

A few researchers were interested in utilizing cooking palm oil as a carbon precursor to synthesized graphene. A few graphene layers were successfully synthesized from palm oil using a single-zone furnace of the CVD method, placed inside an alumina boat beside Ni foil ${ }^{[35]}$. At such a high growth temperature between $800^{\circ} \mathrm{C}-1000^{\circ} \mathrm{C}$, palm oil is estimated to be thermally decomposed and diffused onto the metal substrate. The graphene is formed due to the carbon atoms' segregation and precipitation on the Ni surface during the cooling period. The feasibility of palm oil to synthesizing graphene has shown a promising method when the growth conditions utilize a low precursor. A high volume of palm oil leads to the formation of amorphous carbon and induced graphite sedimentation. The graphene films exhibit surface color with a mixture of light blue, yellow and purple which indicates their structures. The yellowish surface shows thick graphite, while light blue indicates this graphite. The purple color indicates multilayer graphene. The graphene layer's quality can be obtained from ratios of $I_{D} / I_{G}$ and $I_{2 D} / I_{G}$ from Raman studies. The lowest $\mathrm{I}_{2 \mathrm{D}} / \mathrm{I}_{\mathrm{G}}$ is 1.1 with $\mathrm{FWHM}$ of the $2 \mathrm{D}$ band is $45 \mathrm{~cm}^{-1}$. Salifairus et al. reported that low $I_{D} / I_{G}$ and $I_{2 D} / I_{G}$ values of 0.27 and 1.19 , respectively for graphene layers growth at $900^{\circ} \mathrm{C}$ possesses good quality ${ }^{[36]}$. This result is due to nucleation that appeared primarily on the step edge, while nucleation at the terrace turns out to be substantial at a relatively high temperature and on a smooth surface, due to the low barrier energy. In addition, the percentage of reflectance as confirmed by UV-vis 
spectra is less than $30 \%$ between 266 and $285 \mathrm{~nm}$. Interestingly, the increment of reflectance percentage is reflected by increases in deposition temperature. Despite optimizing the ambient furnace temperature for graphene growth, different vaporization temperatures for palm oil have also been studied ${ }^{[37]}$. Various vaporization temperatures ranging from $250^{\circ} \mathrm{C}$ to $450^{\circ} \mathrm{C}$ showed a significant effect on the carbon atoms' generation rate while maintaining the growth temperature at $900^{\circ} \mathrm{C}$ using a double furnace. From the study, high temperature $\left(\sim 450^{\circ} \mathrm{C}\right)$ of precursor lead to thick amorphous carbon film on the Ni surface due to the high energy for the diffusion of carbon atoms. Likewise, graphene growth at $350^{\circ} \mathrm{C}$ possesses multilayer graphene, which is confirmed by Raman studies.
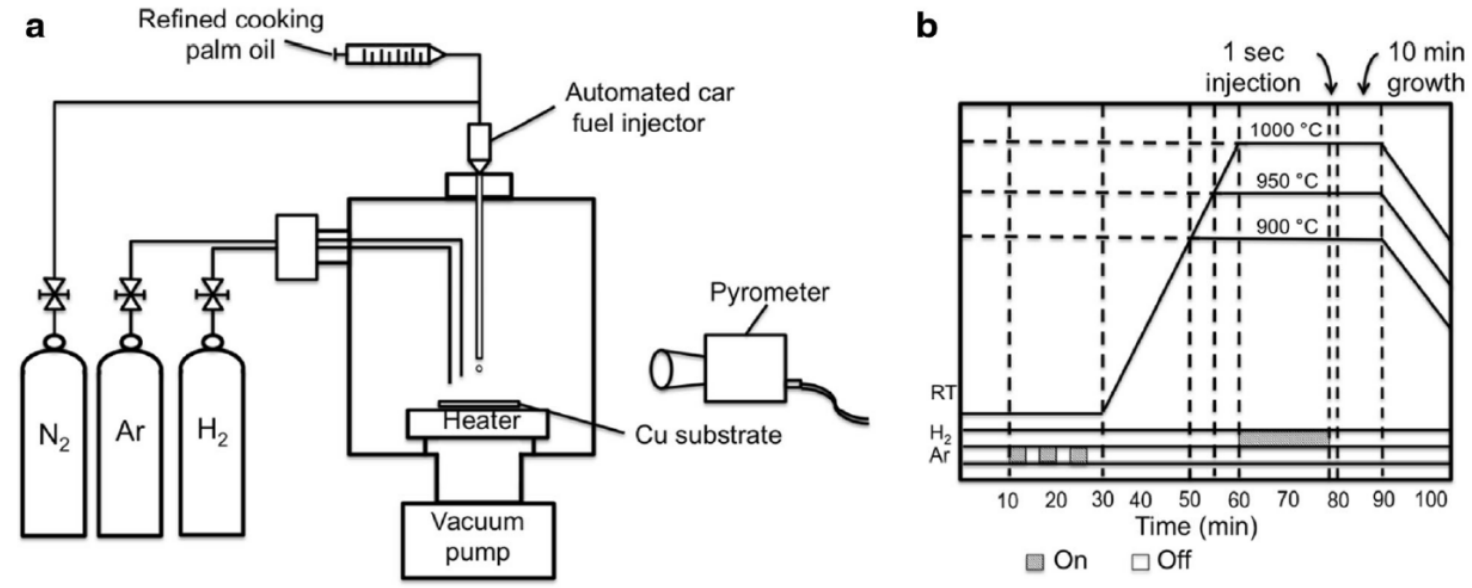

Figure 3. (a) a schematic diagram for setting up a homemade spray-injector CVD and (b) growth chart. Adapted from ref. [38].

Maarof et al. studied graphene properties on a copper substrate using a refined cooking palm oil by a homemade spray injector-assisted chemical vapor deposition system ${ }^{[38]}$ Large single-layer graphene was obtained on the copper substrates up to $97 \%$ coverage area from a size $6400 \mu m^{2}$. A 2D band from Raman spectra reveals $10 \mathrm{~cm}^{-1}$ for FWHM values indicating relatively high crystallinity graphene films. In addition, $I_{D} / I_{G}$ ratio for all samples is mainly in the range of $0.5-1.3$. Although the single-layer graphene crystallinity is relatively good, as confirmed by Raman studies, the concentration of defects remains relatively large. Therefore, the authors suggest having a flash-cooling technique immediately after the graphene growth. The properties of Cu possess low carbon solubility; thus, the Cu metal catalyst results in a surface-mediated mechanism. The high carbon concentration medium leads to a large amount of decomposed $\mathrm{C}$ that keeps depositing onto the $\mathrm{Cu}$ surface to form bilayer graphene (BLR) and multilayer graphene (MLG). However, the results from the flash-cooling method need further studies to prove this statement. Figure 3 shows a diagram for a homemade spray-assisted CVD setup and growth chart. The nozzle distance relative to the substrates implies that the shortest distance $(\mathrm{d}=1 \mathrm{~cm})$ is the excellent distance from the longest distance $(d=6 \mathrm{~cm})$, which leads to the formation of amorphous carbon and scratches on the $\mathrm{Cu}$ substrate surface. In addition, the graphene structure grown at $d=1$ $\mathrm{cm}$ dominated by single-layer and $\mathrm{d}=6 \mathrm{~cm}$ dominated by a multilayer structure. These results are interpreted from the ratio of $I_{2 D} / I_{G}$ as the following value: more than 2 as single layer; in between 1 and 2 as bilayer; and less than 1 as multilayer ${ }^{[39]}$.

\section{Discussion and Perspective}

With considerable progress over the years, various problems must be addressed to fully realize their potential for a wide range of graphene applications. A significant challenge of graphene growth using liquid carbon precursor is proper synthesis procedure control and a comprehensive understanding of CVD growth mechanisms under different conditions. Controlling the coverage area of graphene films and the number of layers is a major challenge as well. Further advancement of catalyst-free or metal- 
free methods is also important to extend the potential of graphene in electronic applications. Table 1 summarized the synthesized graphene method with the liquid precursor, its catalyst, its graphene yield, and $I_{D} / I_{G}$ ratio from Raman studies. Although liquid source has successfully proven to grow graphene films on metallic substrates, most reported catalysts only use Cu foil. Therefore, it is important to explore alternative catalysts or substrates that are compatible with the CVD technique. In addition, Raman characterization needs to be fully utilized to study $I_{D} / I_{G}$ ratio and FWHM of a $2 \mathrm{D}$ peak to compare its characteristics with the established CVD method using gas precursor [40][41]. Moreover, there is still plenty of room to develop a proper transfer method to remove graphene film from their substrates onto another desirable surface while retaining its quality.

Table 1. A summary of liquid carbon precursor, its synthesized method, and properties.

\begin{tabular}{|c|c|c|c|c|c|c|}
\hline Precursor & Method & Catalyst & $\begin{array}{c}\text { Growth } \\
\text { temperature }\left({ }^{\circ} \mathrm{C}\right)\end{array}$ & Graphene Yield & $\mathrm{I}_{\mathrm{D}} / \mathrm{I}_{\mathrm{G}}$ ratio & Ref. \\
\hline $\begin{array}{l}\text { ethanol and } \\
\text { pentane }\end{array}$ & $\begin{array}{c}\text { Atmospheric } \\
\text { pressure CVD } \\
\text { (APCVD) }\end{array}$ & Cu foil & 900 & $\begin{array}{c}50-60 \% \text { single and } \\
\text { double layer }\end{array}$ & 0.23 & [17] \\
\hline ethanol & CVD & Ni foil & 900 & single layer & - & [16] \\
\hline ethanol & CVD & $\begin{array}{c}\text { Cu foil - with } \\
\text { oxidation treatment }\end{array}$ & 1065 & $\begin{array}{l}\text { graphene flakes - } \\
\text { single crystal }\end{array}$ & $>10$ & [19] \\
\hline $\begin{array}{c}2- \\
\text { phenylethanol } \\
\text { and ethanol }\end{array}$ & APCVD & Cu foil & $980-990$ & $\begin{array}{l}\text { mono-, bi- and tri- } \\
\text { layer }\end{array}$ & $0.1-0.5$ & [18] \\
\hline ethanol & CVD & $\begin{array}{l}\text { pre-oxidation } \\
\text { treatment of } \mathrm{Cu} \text { foil }\end{array}$ & $1000-1070$ & $\begin{array}{l}\text { graphene island } \\
\text { single crystal }\end{array}$ & - & [20] \\
\hline ethanol & thermal CVD & stainless steel & 850 & $\begin{array}{c}\text { multilayer, single, bi-, } \\
\text { tri-layer }\end{array}$ & - & {$[21]$} \\
\hline hexane & $\begin{array}{c}\text { Vacuum- } \\
\text { assisted CVD }\end{array}$ & Cu foil & 950 & $\begin{array}{c}\text { Monolayer and } \\
\text { double layer }\end{array}$ & - & [22] \\
\hline hexane & CVD & Cu foil & 1000 & $\begin{array}{l}\text { multilayer (7 - } 11 \\
\text { layers) }\end{array}$ & - & [23] \\
\hline benzene & $\begin{array}{l}\text { Low-pressure } \\
\text { CVD }\end{array}$ & Cu foil & $300-500$ & Monolayer flakes & - & [24] \\
\hline benzene & APCVD & Cu foil & 300 & monolayer & 0.35 & [25] \\
\hline benzene & APCVD & Cu foil & $100-300$ & monolayer & $0.1-0.2$ & [26] \\
\hline benzene & CVD & Cu foil & $800-1000$ & Bi- and few-layer & $1-8$ & {$[28]$} \\
\hline benzene & $\begin{array}{c}\text { Low-pressure } \\
\text { CVD }\end{array}$ & Cu foil & $400-1000$ & Multilayer & $0.08-0.15$ & [29] \\
\hline toluene & two step CVD & $\begin{array}{c}\text { electropolished cu } \\
\text { foil }\end{array}$ & $300-600$ & monolayer & $<0.85$ & [30] \\
\hline $\begin{array}{c}\text { toluene and } \\
\text { xylene }\end{array}$ & CVD & $\mathrm{SiC}$ & 900 & Few layers & - & [31] \\
\hline $\begin{array}{l}\text { Melaleuca } \\
\text { alternifolia oil }\end{array}$ & PECVD & Free catalyst & 800 & 3D nanostructured & 0.63 & [33] \\
\hline $\begin{array}{l}\text { Melaleuca } \\
\text { alternifolia oil }\end{array}$ & PECVD & Ni foam & 800 & Vertical nanosheet & $1.05-1.59$ & [34] \\
\hline Soybean oil & CVD & Ni foil & 800 & Single to multi-layers & $0.15-0.25$ & [32] \\
\hline Palm oil & CVD & Ni sheet & $800-1100$ & Multilayer & 0.27 & [36] \\
\hline Palm oil & $\begin{array}{c}\text { Thermal CVD } \\
\text { (TCVD) }\end{array}$ & Ni foil & $800-1000$ & Few layers & - & [35] \\
\hline Palm oil & Double TCVD & Ni foil & 900 & Multilayer & - & [37] \\
\hline Palm oil & $\begin{array}{c}\text { Spray injector } \\
\text { CVD }\end{array}$ & Cu foil & $900-1000$ & Single-layer & $0.5-1.3$ & [38] \\
\hline
\end{tabular}


Undoubtedly, CVD techniques for graphene synthesis are a promising method for large-scale industrial applications. However, the use of liquid precursor for practical applications are still scarce as wrinkle, impurities and defect are always observed after the graphene transfer process. Therefore, sufficient theoretical understanding with a systematic experimental investigation is needed to overcome this shortcoming. In addition, a critical study is desired, should either deposit graphene directly on target substrates without the need for a transfer process or establish a simple, environmentally friendly method to transfer graphene to target substrates. The direct synthesis of graphene onto desired substrates is crucial for practical applications. Moreover, it could be a grand task to improve the CVD approach to directly synthesize graphene on polymer substrates for flexible-based devices. This is likely a new area and development for the future. Besides, an extensive study on the Fermi level could be considered for future studies as this knowledge is highly important for any optical and electronic application.

Another interesting study shows that a reduction in growth temperature using liquid carbon precursor is opening a new frontier in graphene synthesis. To date, most of the literature reported ethanol and benzene as a carbon source for graphene growth. The popularity of benzene increases because of its effectiveness and feasibility of low growth temperature and low-cost graphene production. Moreover, a reduction in growth temperature as low as $300^{\circ} \mathrm{C}$ may lessen graphene CVD cycle time. However, some of the mentioned liquid carbon precursors are carcinogenic to humans. Therefore, researchers are looking for other alternatives to carbon sources to overcome these drawbacks.

As was highlighted, the use of green liquid precursor is opening a new way in the graphene synthesis route compared to conventional CVD growth. As has been demonstrated in this review, various factors influencing as-produced graphene film quality include process setup, pressure, the temperature of growth, and carbon sources. However, most of the reported works only presented the carbon source's novelty and the experimental setup's uniqueness. Knowing the effect of the growth conditions and their relationship with the physical properties still lacks fundamental understanding. The correlation between the liquid carbon source with other common metal substrates also needs to be thoroughly investigated. Although progress in green liquid precursor graphene synthesis techniques has shown the potential to develop uniform, single-crystalline monolayer graphene, further research is needed to explore the possibility of exploring very low growth temperature and transfer-free graphene process. Moreover, the graphene growth productivity, such as the ability to reproduce the films, must be improved before practical applications. Carbon waste graphene synthesis was limited to laboratory size. Their yield is also far below a commercialized level. Therefore, the possibility of depositing precursors uniformly over a large area is recommended.

The introduction of plasma in synthesizing graphene from gas precursor have been proven to promote a better quality of the as-produced films at low temperature. Researchers should also use this latest technology for liquid carbon precursors. Moreover, to lessen cost and enhance graphene transfer quality, it is advisable to synthesize graphene on the target substrates or new safe transfer technology. Other than that, the researcher should also simplify the experimental setup and equipment for graphene growth. Further production of graphene from carbon-organic waste should also be considered to improve the efficiency of graphene for real applications.

\section{Conclusions}

With its remarkable properties including high specific surface area and excellent chemical and thermal stability, graphene has gained much attention, facilitating applications in various fields. A detailed synthesis of large-area graphene using liquid carbon precursors via the CVD technique has been reviewed in this article. Various liquid precursor has been demonstrated to produce graphene layers, and it is proven to be alternative for gas precursor. However, constraints such as the high cost of graphene growth must be addressed to commercialization in various applications. 


\section{Conflicts of interest}

The authors declare that there is no conflict of interest regarding the publication of this paper

\section{Funding statement}

The authors are grateful to the Universiti Teknologi Malaysia under research grant R.K130000.7340.4L825 and Universiti Kuala Lumpur under short term research grant STR19062 for financial research support.

\section{References}

[1] A. K. Geim and K. S. Novoselov, "The rise of graphene," Nat. Mater., vol. 6, no. 3, pp. 183-191, Mar. 2007.

[2] R. K. Singh Raman et al., "Protecting copper from electrochemical degradation by graphene coating," Carbon N. Y., vol. 50, no. 11, pp. 4040-4045, Sep. 2012.

[3] M.-S. Kim, J.-M. Woo, D.-M. Geum, J. R. Rani, and J.-H. Jang, "Effect of copper surface pre-treatment on the properties of CVD grown graphene," AIP Adv., vol. 4, no. 12, p. 127107, Dec. 2014.

[4] T. J. Gnanaprakasa et al., "The role of copper pretreatment on the morphology of graphene grown by chemical vapor deposition," Microelectron. Eng., vol. 131, pp. 1-7, Jan. 2015.

[5] R. Addou, A. Dahal, P. Sutter, and M. Batzill, "Monolayer graphene growth on Ni (111) by low temperature chemical vapor deposition," Appl. Phys. Lett., vol. 100, no. 2, p. 021601, Jan. 2012.

[6] E. Ozceri and Y. Selamet, "Influence of buffer layers on Ni thin film structure and graphene growth by CVD," $J$. Phys. D. Appl. Phys., vol. 48, no. 45, p. 455302, Nov. 2015.

[7] G. W. Cushing, V. Johánek, J. K. Navin, and I. Harrison, "Graphene Growth on Pt(111) by Ethylene Chemical Vapor Deposition at Surface Temperatures near 1000 K," J. Phys. Chem. C, vol. 119, no. 9, pp. 4759-4768, Mar. 2015

[8] T. Gao et al., "Growth and Atomic-Scale Characterizations of Graphene on Multifaceted Textured Pt Foils Prepared by Chemical Vapor Deposition," ACS Nano, vol. 5, no. 11, pp. 9194-9201, Nov. 2011.

[9] E. Sutter, P. Albrecht, and P. Sutter, "Graphene growth on polycrystalline Ru thin films," Appl. Phys. Lett., vol. 95, no. 13, p. 133109, Sep. 2009.

[10] S. Nie et al., "Growth from Below: Graphene Bilayers on $\operatorname{Ir}(111), "$ ACS Nano, vol. 5, no. 3, pp. 2298-2306, Mar. 2011.

[11] M. A. Azam et al., "Review-Critical Considerations of High Quality Graphene Synthesized by PlasmaEnhanced Chemical Vapor Deposition for Electronic and Energy Storage Devices," ECS J. Solid State Sci. Technol., vol. 6, no. 6, pp. M3035-M3048, Jan. 2017.

[12] C.-M. Seah, S.-P. Chai, and A. R. Mohamed, "Mechanisms of graphene growth by chemical vapour deposition on transition metals," Carbon N. Y., vol. 70, pp. 1-21, Apr. 2014.

[13] M. Marchena, D. Janner, T. L. Chen, V. Finazzi, and V. Pruneri, "Low temperature direct growth of graphene patterns on flexible glass substrates catalysed by a sacrificial ultrathin Ni film," Opt. Mater. Express, vol. 6, no. 8, p. 2487, Aug. 2016.

[14] I. Vlassiouk et al., "Electrical and thermal conductivity of low temperature CVD graphene: the effect of disorder," Nanotechnology, vol. 22, no. 27, p. 275716, Jul. 2011.

[15] Y. Gong et al., "Layer-Controlled and Wafer-Scale Synthesis of Uniform and High-Quality Graphene Films on a Polycrystalline Nickel Catalyst," Adv. Funct. Mater., vol. 22, no. 15, pp. 3153-3159, Aug. 2012.

[16] Y. Miyata, K. Kamon, K. Ohashi, R. Kitaura, M. Yoshimura, and H. Shinohara, "A simple alcohol-chemical vapor deposition synthesis of single-layer graphenes using flash cooling," Appl. Phys. Lett., vol. 96, no. 26, p. 263105, Jun. 2010.

[17] X. Dong et al., "Growth of large-sized graphene thin-films by liquid precursor-based chemical vapor deposition under atmospheric pressure," Carbon N. Y., vol. 49, no. 11, pp. 3672-3678, Sep. 2011.

[18] J. Campos-Delgado et al., "CVD synthesis of mono- and few-layer graphene using alcohols at low hydrogen concentration and atmospheric pressure," Chem. Phys. Lett., vol. 584, pp. 142-146, Oct. 2013.

[19] X. Chen et al., "Chemical vapor deposition growth of $5 \mathrm{~mm}$ hexagonal single-crystal graphene from ethanol," Carbon N. Y., vol. 94, pp. 810-815, Nov. 2015.

[20] A. Gnisci et al., "Ethanol-CVD Growth of Sub-mm Single-Crystal Graphene on Flat Cu Surfaces," J. Phys. Chem. C, vol. 122, no. 50, pp. 28830-28838, Dec. 2018.

[21] R. John, A. Ashokreddy, C. Vijayan, and T. Pradeep, "Single- and few-layer graphene growth on stainless steel substrates by direct thermal chemical vapor deposition," Nanotechnology, vol. 22, no. 16, p. 165701, Apr. 2011

[22] A. Srivastava et al., "Novel Liquid Precursor-Based Facile Synthesis of Large-Area Continuous, Single, and Few-Layer Graphene Films," Chem. Mater., vol. 22, no. 11, pp. 3457-3461, Jun. 2010.

[23] C. B. Flores and D. M. López, "Multilayer Graphene Synthesized by CVD Using Liquid Hexane as the Carbon Precursor," World J. Condens. Matter Phys., vol. 01, no. 04, pp. 157-160, 2011.

[24] Z. Li et al., "Low-Temperature Growth of Graphene by Chemical Vapor Deposition Using Solid and Liquid Carbon Sources," ACS Nano, vol. 5, no. 4, pp. 3385-3390, Apr. 2011.

[25] C. Kang, D. H. Jung, and J. S. Lee, "Atmospheric Pressure Chemical Vapor Deposition of Graphene Using a 
Liquid Benzene Precursor," J. Nanosci. Nanotechnol., vol. 15, no. 11, pp. 9098-9103, Nov. 2015.

[26] J. Jang et al., "Low-temperature-grown continuous graphene films from benzene by chemical vapor deposition at ambient pressure," Sci. Rep., vol. 5, no. 1, p. 17955, Dec. 2015.

[27] Y. Xue et al., "Low Temperature Growth of Highly Nitrogen-Doped Single Crystal Graphene Arrays by Chemical Vapor Deposition," J. Am. Chem. Soc., vol. 134, no. 27, pp. 11060-11063, Jul. 2012.

[28] R. Shanmugam, M. Rangarajan, S. Devanathan, V. G. Sathe, R. Senthilkumar, and N. K. Kothurkar, "A design of experiments investigation of the effects of synthesis conditions on the quality of CVD graphene," Mater. Res. Express, vol. 3, no. 12, p. 125601, Dec. 2016

[29] M. H. Khan et al., "Hydrogen sieving from intrinsic defects of benzene-derived single-layer graphene," Carbon N. Y., vol. 153, pp. 458-466, Nov. 2019.

[30] B. Zhang et al., "Low-temperature chemical vapor deposition growth of graphene from toluene on electropolished copper foils," ACS Nano, vol. 6, no. 3, pp. 2471-2476, 2012.

[31] J. G. Kim, W. S. Kim, Y. H. Kim, C. H. Lim, and D. J. Choi, "Formation of graphene on SiC by chemical vapor deposition with liquid sources," Surf. Coatings Technol., vol. 231, pp. 189-192, Sep. 2013.

[32] D. H. Seo et al., "Single-step ambient-air synthesis of graphene from renewable precursors as electrochemical genosensor," Nat. Commun., vol. 8, no. 1, p. 14217, Apr. 2017.

[33] M. V. Jacob et al., "Catalyst-Free Plasma Enhanced Growth of Graphene from Sustainable Sources," Nano Lett., vol. 15, no. 9, pp. 5702-5708, Sep. 2015.

[34] B. Ouyang, Y. Zhang, Z. Zhang, H. J. Fan, and R. S. Rawat, "Green synthesis of vertical graphene nanosheets and their application in high-performance supercapacitors," RSC Adv., vol. 6, no. 28, pp. 23968-23973, 2016.

[35] S. F. A. Rahman, M. R. Mahmood, and A. M. Hashim, "Growth of graphene on nickel using a natural carbon source by thermal chemical vapor deposition," Sains Malaysiana, vol. 43, no. 8, pp. 1205-1211, 2014.

[36] M. J. Salifairus, S. B. Abd Hamid, T. Soga, S. A. H. Alrokayan, H. A. Khan, and M. Rusop, "Structural and optical properties of graphene from green carbon source via thermal chemical vapor deposition," J. Mater. Res., vol. 31, no. 13, pp. 1947-1956, 2016.

[37] M. Robaiah, M. Rusop, S. Abdullah, Z. Khusaimi, H. Azhan, and N. A. Asli, "Synthesis graphene layer at different waste cooking palm oil temperatures," in AIP Conference Proceedings, 2017, p. 030008.

[38] S. Maarof, A. A. Ali, and A. M. Hashim, "Synthesis of Large-Area Single-Layer Graphene Using Refined Cooking Palm Oil on Copper Substrate by Spray Injector-Assisted CVD," Nanoscale Res. Lett., vol. 14, no. 1, p. 143 Dec. 2019

[39] L. M. Malard, M. A. Pimenta, G. Dresselhaus, and M. S. Dresselhaus, "Raman spectroscopy in graphene," Phys. Rep., vol. 473, no. 5-6, pp. 51-87, Apr. 2009.

[40] J. Wang et al., "A review of graphene synthesisatlow temperatures by CVD methods," New Carbon Mater., vol. 35, no. 3, pp. 193-208, Jun. 2020.

[41] S. Naghdi, K. Y. Rhee, and S. J. Park, "A catalytic, catalyst-free, and roll-to-roll production of graphene via chemical vapor deposition: Low temperature growth," Carbon N. Y., vol. 127, pp. 1-12, Feb. 2018. 